Bull.Fac, Agric.,Cairo Univ.,61:346-354 (2010).

\title{
GENETIC ANALYSIS OF DIALLEL CROSSES BETWEEN FIVE MODERATELY MATURING INBRED LINES OF MAIZE
}

(Received: 25. 3. 2010)

\author{
By \\ M. A. Hussain and I. H. Ali * \\ Agriculture College, Duhok University, Iraq and* Agriculture College, Salahaddin University, Iraq
}

\begin{abstract}
This study was conducted during the spring season of 2008 at the Farm of the College of Agriculture - Duhok University, Iraq by using five maize inbred lines locally developed. Half diallel cross design was applied for these inbred lines to produce ten $F_{1}$ single crosses. A yield trial for the 10 hybrids and their parents was carried out in the Research Farm of the College of Agriculture Salahaddin University, Iraq using a randomized complete block design with three replications in order to study the gene action for yield and yield components beside estimating heterosis, combining ability and variance components (additive, dominance and environmental variance) in addition to estimate some other genetic parameters. The results showed the presence of significant differences among genotypes for all characters. For mean grain yield the best parent was ( $\mathrm{ZP}-197)$, while the best hybrid was ( $\mathrm{ZP}-707 \times \mathrm{DK})$. The parent $(\mathrm{ZP}-595)$ appeared to be the best general combiner for most studied characters, while the hybrid $(\mathrm{ZP}-607) \times(\mathrm{ZP}-707)$ appeared to be the best for specific combining ability. Ratio of general to specific combining ability variances was more than one for all characters, indicating that the characters were under the effect of over dominance. Broad sense heritability value was found to be high in all the studied characters, while the narrow sense heritability was moderate for silking and tasselling date, grain yield / plant, number of kernels / row , 100- kernel weight and low for plant height, ear height , leaf area , number of rows / ear, ear length and number of kernels / ear. The expected genetic advance from selection was high for grain yield / plant and 100- kernel weight. The hybrids (DK $\times$ $\mathrm{ZP}-197)$ and (ZP $-707 \times \mathrm{ZP}-197)$ gave the highest heterosis value in silking and tasselling date, while the heterosis value was high for the flag leaf area, ear height and grain yield / plant and was low for kernels / row , rows / ear, ear length and 100- kernel weight with different hybrids.
\end{abstract}

Key words: combining ability, diallel cross, genetic parameters, heterosis, maize.

\section{INTRODUCTION}

Maize is the third most important cereal food crops of the world after wheat and rice. It is a multipurpose crop which provides edible oil for human use, feed for poultry and fodder for livestock. In fact, maize has been subjected to extensive genetic studies than any other crop (Hallauer and Miranda, 1988). The development of high yielding hybrids is one of the important objectives of plant breeders. The inbred lines should be evaluated according to general and specific combining ability and selecting the best of them to be entered in hybrid for achieving the high yield. Hybridization is considered one of the most important programs which is used for obtaining superior hybrids for local environment. Such programs need testing the combining ability of inbred lines that are used as parents according to the general combining ability and then selecting the best hybrid combination for specific combing ability for yield (Ahmed and Ali, 2003). Half diallel cross method is considered one of the effective ways for estimating the general and specific combining ability to select the best parents of inbred lines. The first who used diallel cross was Sparague and Tatum (1942) working on maize. The concept of diallel cross was laid by Griffing (1956) and described by Singh and Chaudhary (2007). Many researchers and plant breeders used the inbred lines of maize in diallel cross analysis(Ojo et al., 2001; Al-Sweediy, 2002; Reza'ei et al.,2004; Al-Jamili ,2006 ; Chungji et al., 2006; Rather et al., 2007 and Rather et al., 2009). Many studies were conducted 
concerning heritability for characters in different crops, Deletic et al., (2005) and Najeeb et al., (2009) obtained high heritability for the most characters, while Cook and Hallauer and Miranda (1988) and Dawod et al. (2009) showed that the average dominance was less than one for all characters except the grain yield/plant, which reached 1.7. Paterniani et al. (2004) found that the best expected genetic advance in grain yield reached $7.9 \%$. Lee et al. (2006); Chungji et al. (2006) and Dawod et al. (2009) obtained high heterosis for plant height, ear height and grain yield/plant.

The objective of this study was to estimate some genetic parameters and heterotic effect and to determine suitable inbred parents and promising crosses for grain yield by using half diallel cross design between five inbred lines of corn.

\section{MATERIALS AND METHODS}

The diallel crosses were carried out at the experimental field of the College of Agriculture, Duhok University, Iraq during the spring season 2008. Five inbred lines of corn were used [(1) ZP-607, (2) ZP-707, (3) DK, (4) ZP-197, and (5) ZP-595]. The inbred lines were sown on April; each inbred was planted in two rows of $4 \mathrm{~m}$ length, $75 \mathrm{~cm}$ between rows and $25 \mathrm{~cm}$ between plants (Al-Falahi, 2000). A weight of $600 \mathrm{~kg} / \mathrm{ha}$ of compound fertilizer (N. P. K.) (27-27-0) was applied during land preparation. Also, $200 \mathrm{~kg} /$ ha Urea fertilizer $(46 \% \mathrm{~N})$ were added in two doses , the first dosage were added after 30 days from planting and the second was added at anthesis stage. A half diallel crossing program was applied to produce ten single crosses. During the spring season (on April 21, 2009) ten hybrids and five parents were planted at the experimental field of the College of Agriculture, Salahaddin University, Iraq. A randomized complete block design (RCBD) with three replicates was applied. Each replicate consisted of 15 rows ( 5 for parents and ten for hybrids). All recommended cultural practices were done at the same spring season. The data were recorded on days to $50 \%$ tasselling and 50\% silking, plant height $(\mathrm{cm})$, ear height $(\mathrm{cm})$, flag leaf area $\left(\mathrm{cm}^{2)}\right.$, ear length $(\mathrm{cm})$, kernels/row, rows/ear, 100- kernel weight $(\mathrm{g})$, kernels/ear and grain yield/plant (g).The data were analysed by using RCBD design according to Al-Rawi and Khalaf -
Allah (1980). L.S.D was used to compare the means of genotypes. The genetic analysis was based on Griffing's method 2 - fixed model to determine the variance and effects of general and specific combining ability, additive, dominance and environmental variance, average degree of dominance. Heritabilities in broad and narrow sense were determined. Expected genetic advance in absolute and percentage was calculated. Heterosis was estimated as a deviation of $F_{1}$ from the mid parent and high parent values.

\section{RESULTS AND DISCUSSION}

The data in Table (1) show significant mean squares due to genotypes for all studied characters at level 0.01 except for plant height where the significance was at level 0.05 . Table (2) presents the means of genotypes (five inbreds and ten hybrids). The results in Table (2) indicate that inbreds (4) and (5) were shorter than others in plant height $(120 \mathrm{~cm})$ and the inbred (3) was the tallest one $(151.66 \mathrm{~cm})$. The hybrid $(3 \times 5)$ was the tallest hybrid $(161.6$ $\mathrm{cm})$ whereas hybrid $(3 \times 5)$ was the shortest in plant height $(130.0 \mathrm{~cm})$. It can be noticed that inbred (2) was the earliest in days to $50 \%$ tasselling while inbred (4) could be considered the latest (73 days). The lowest value in this character (65 days) was found in the hybrid $(3 \times 4)$, while the hybrid $(1 \times 3)$ was the most delayed genotype in days to $50 \%$ tasselling and the inbreds 2 and 4 were the earliest and latest in days to $50 \%$ silking, respectively. The largest flag leaf area was found in inbred (3) $\left(693.33 \mathrm{~cm}^{2}\right)$, whereas the lowest leaf area was that of inbred $(5)\left(550 \mathrm{~cm}^{2}\right)$. The hybrid $(1 \times 5)$ gave the largest flag leaf area $\left(716.66 \mathrm{~cm}^{2}\right)$, while the lowest one was observed in hybrid $(1 \times 4)\left(610 \mathrm{~cm}^{2}\right)$. The inbred line (3) scored a high ear height $(71.66 \mathrm{~cm})$, while the inbred line (5) gave the lowest ear height $(58.33 \mathrm{~cm})$. The hybrid $(1 \times 5)$ showed the largest ear height $(82.33 \mathrm{~cm})$, while hybrid $(1 \times 2)$ scored the lowest ear height $(64.33 \mathrm{~cm})$. High number of kernels/row was found in inbred (2) (37.00), while the inbred line (5) showed the lowest kernels/row (25.66). The hybrid $(2 \times 5)$ showed the highest number of this trait (40.33), whereas the lowest kernels /ear were observed in hybrid $(1 \times 4)$ (31.66). The largest number of rows/ear was found in line (1) (18), whereas the lowest value of this trait was obtained in line (4) (14). For hybrids, the hybrid $(1 \times 3)$ 
Table (1) : Mean squares for the combining ability effects.

\begin{tabular}{|c|c|c|c|c|c|c|c|c|c|c|c|}
\hline S.O.V. & $\begin{array}{c}\text { Plant } \\
\text { height } \\
\text { (cm) }\end{array}$ & $\begin{array}{c}\text { Days to } 50 \\
\% \\
\text { tasselling } \\
\end{array}$ & $\begin{array}{l}\text { Days to } \\
50 \% \\
\text { silking }\end{array}$ & $\begin{array}{c}\text { Flag leaf } \\
\text { area } \\
\left(\mathrm{cm}^{2}\right)\end{array}$ & $\begin{array}{c}\text { Ear } \\
\text { height } \\
(\mathbf{c m})\end{array}$ & $\begin{array}{c}\text { Grain yield } \\
\text { /plant } \\
\text { (g) }\end{array}$ & $\begin{array}{c}\text { Kernels / } \\
\text { row }\end{array}$ & $\begin{array}{c}\text { Rows/ } \\
\text { ear }\end{array}$ & $\begin{array}{c}\text { Ear } \\
\text { length } \\
(\mathrm{cm})\end{array}$ & $\begin{array}{c}100 \\
\text { Kernel } \\
\text { weight }(\mathrm{g}) \\
\end{array}$ & $\begin{array}{l}\text { Kernels } \\
\text { / ear }\end{array}$ \\
\hline Rep. & 1457.22 & 3.28 & 3.28 & 6202.22 & 596.28 & 256.15 & 8.86 & 2.28 & 0.55 & 16.02 & 10002.07 \\
\hline Genotypes & $468.36^{* *}$ & $24.16^{* *}$ & $7.46^{* *}$ & $5968.88^{* * *}$ & $135.23^{*}$ & $2255.74^{* *}$ & $45.19^{* *}$ & $7.27^{* *}$ & $2.74^{* *}$ & $16.94^{* * *}$ & $25426.7^{* *}$ \\
\hline G.C.A. & $496.61^{* *}$ & $29.39^{* *}$ & $8.19^{* *}$ & $7215.71^{* *}$ & $128.65^{*}$ & $4750.19^{* *}$ & $61.19^{* *}$ & $5.84^{* *}$ & $2.92^{* *}$ & $34.29^{* *}$ & $21526.23^{* * *}$ \\
\hline S.C.A. & $457.06^{* *}$ & $22.07^{* *}$ & $7.16^{* *}$ & $5470.15^{* *}$ & $137.86^{*}$ & $1257.96^{* * *}$ & $38.79^{* *}$ & $7.85^{* *}$ & $2.67^{* *}$ & $10.00^{* *}$ & $26986.9^{* * *}$ \\
\hline Error & 35.28 & 0.76 & 0.60 & 1044.39 & 31.49 & 198.38 & 5.47 & 0.88 & 0.47 & 2.31 & 3148.46 \\
\hline
\end{tabular}

*and ** indicate significant at 0.05 and 0.01 probability levels, respectively.

Table (2): Mean performance of parents and hybrids for the studied characters.

\begin{tabular}{|c|c|c|c|c|c|c|c|c|c|c|c|}
\hline Genotypes & $\begin{array}{c}\text { Plant } \\
\text { height } \\
(\mathbf{c m})\end{array}$ & $\begin{array}{c}\text { Days to } 50 \\
\% \\
\text { tasselling }\end{array}$ & $\begin{array}{c}\text { Days to } 50 \\
\% \\
\text { silking }\end{array}$ & $\begin{array}{c}\text { Flag leaf } \\
\text { area } \\
\left(\mathrm{cm}^{2)}\right.\end{array}$ & $\begin{array}{c}\begin{array}{c}\text { Ear } \\
\text { height } \\
(\mathrm{cm})\end{array}\end{array}$ & $\begin{array}{c}\text { Grain yield } \\
\text { /plant } \\
\text { (gm) }\end{array}$ & $\begin{array}{c}\text { Kernels / } \\
\text { row }\end{array}$ & $\begin{array}{c}\text { Rows/ } \\
\text { ear }\end{array}$ & $\begin{array}{c}\text { Ear } \\
\text { length } \\
(\mathrm{cm})\end{array}$ & $\begin{array}{c}100 \\
\text { Kernel } \\
\text { weight }(\mathrm{g})\end{array}$ & $\begin{array}{c}\text { Kernels } \\
\text { / ear }\end{array}$ \\
\hline 1 & 145.00 & 67.66 & 70.33 & 683.33 & 65.00 & 115.33 & 33.33 & 18.00 & 15.33 & 19.66 & 600.00 \\
\hline 2 & 125.00 & 68.00 & 67.33 & 616.66 & 60.00 & 136.66 & 37.00 & 15.00 & 15.00 & 23.66 & 592.00 \\
\hline 3 & 151.66 & 67.33 & 69.66 & 693.33 & 71.66 & 104.66 & 33.00 & 16.00 & 15.00 & 20.33 & 527.00 \\
\hline 4 & 120.00 & 73.00 & 76.00 & 596.66 & 68.33 & 74.00 & 31.33 & 14.00 & 14.00 & 18.00 & 480.00 \\
\hline 5 & 120.00 & 70.00 & 72.00 & 550.00 & 58.33 & 66.66 & 25.66 & 14.66 & 13.66 & 19.00 & 372.66 \\
\hline $1 \times 2$ & 145.00 & 67.66 & 69.66 & 623.33 & 64.33 & 160.33 & 36.00 & 16.66 & 17.00 & 26.33 & 614.66 \\
\hline $1 \times 3$ & 151.00 & 68.66 & 71.00 & 683.33 & 76.66 & 149.66 & 33.66 & 19.33 & 16.33 & 23.00 & 644.00 \\
\hline $1 \times 4$ & 145.00 & 67.33 & 69.33 & 610.00 & 66.66 & 100.66 & 31.66 & 14.00 & 15.00 & 22.00 & 430.66 \\
\hline $1 \times 5$ & 150.00 & 67.33 & 70.33 & 716.66 & 82.66 & 120.00 & 32.33 & 17.33 & 15.00 & 22.33 & 540.00 \\
\hline $2 \times 3$ & 145.00 & 68.00 & 70.66 & 626.66 & 66.66 & 163.33 & 40.00 & 17.33 & 15.66 & 22.00 & 686.00 \\
\hline $2 \times 4$ & 146.60 & 65.33 & 68.00 & 616.66 & 71.66 & 118.66 & 37.66 & 18.00 & 14.66 & 18.33 & 666.00 \\
\hline $2 \times 5$ & 139.00 & 65.66 & 68.00 & 683.33 & 66.66 & 129.33 & 40.33 & 16.00 & 16.66 & 20.33 & 618.66 \\
\hline $3 \times 4$ & 130.00 & 65.00 & 67.33 & 633.33 & 71.66 & 108.66 & 34.33 & 16.66 & 14.66 & 19.66 & 570.66 \\
\hline $3 \times 5$ & 161.60 & 68.33 & 71.00 & 670.00 & 79.00 & 110.66 & 34.33 & 16.66 & 14.33 & 20.33 & 572.66 \\
\hline $4 \times 5$ & 151.60 & 68.33 & 71.00 & 660.00 & 73.33 & 119.66 & 39.33 & 17.66 & 16.00 & 17.66 & 696.00 \\
\hline L.S. D 5\% & 14.29 & 2.10 & 1.86 & 77.74 & 13.44 & 33.88 & 5.62 & 2.26 & 1.6 & 3.66 & 134.97 \\
\hline L.S. D $1 \%$ & 20.72 & 3.04 & 2.71 & 112.74 & 19.57 & 49.14 & 8.16 & 3.29 & 2.41 & 2.31 & 195.76 \\
\hline
\end{tabular}


gave the largest number of rows / ear (19.33); the hybrid $(1 \times 4)$ gave the lowest number for this trait (14). The inbred line (1) exhibited the largest ear length $(15.33 \mathrm{~cm})$, while the shortest ear was observed in line (5) (13.66 $\mathrm{cm})$. For hybrids, the hybrid $(1 \times 2)$ showed the largest ear length $(17.00 \mathrm{~cm})$, while the shortest ear was recorded in hybrid $(3 \times 5)$ $(14.33 \mathrm{~cm})$

The highest mean of 100- kernel weight was observed in inbred (2) (23.66 gm) whereas the lowest mean was obtained in inbred (4) $(18.66 \mathrm{gm})$. The hybrid $(1 \times 2)$ gave the highest kernel weight $(26.33 \mathrm{gm})$, while the lowest grain weight was obtained in hybrid $(4 \times 5)$ $(17.66 \mathrm{gm})$. The inbred line (1) was the best line for number of kernels / ear (600). The lowest for this trait was found in inbred (5), (372.66). For hybrids, the hybrid $(4 \times 5)$ gave the highest number of kernels/ear (696) while the lowest number was found in hybrid $(1 \times 4)$ (430.66). The highest mean grain yield/plant was observed in line (3) (71.66 gm), while the lowest grain yield/plant was recorded in line (5) $(66.66 \mathrm{~g})$. The hybrid $(2 \times 3)$ gave the highest mean grain yield/plant and value was $(163.33 \mathrm{~g})$. The lowest grain yield/plant was found in hybrid $(1 \times 4)(100.66 \mathrm{~g})$. These results agree with the findings of Ojo et al.(2001), AlSweediy (2002), Rezaei et al. (2004)and Rather et al., (2007 and 2009).

Table (3) shows that for plant height trait, the highest positive G.C.A. effect was in parent (3), while the highest positive S.C.A. effect was found in hybrid $(1 \times 2)$. For days to $50 \%$ tasselling the highest negative G.C.A. effect was in parent (3). The highest negative S.C.A. effect for the same trait was found in hybrid $(3 \times 4)$. For days to $50 \%$ silking the highest negative G.C.A. effect was in parent (2), whereas the highest negative S.C.A. effect was in hybrid $(3 \times 4)$. The parent (2) gave high G.C.A. effect for three traits (flag leaf area, yield/plant and kernels/ear), while the hybrid $(1 \times 2)$ had negative S.C.A. effects in traits: flag leaf area, yield /plant, ear length and 100kernel weight. Similar results in maize have been reported by Al-Savie (2005), Derera et al . (2007), Mohammdi et al. (2008) and Rather et al. (2009). Table (4) presents estimates of additive, dominance and environmental variance for the studied characters. The dominance variance was more than additive variance for all characters except the traits:
100- kernel weight and number of kernels/row. This indicates that these traits were under control of the dominance gene effect. These results are in conformity with findings of Hamed (2008) and Rather et al.(2009). The data in Table (5) show the average degree of dominance and heritability in broad and narrow sense. The average degree of dominance was greater than one for all characters, indicating that these traits were under control of overdominance gene effect. High heritability in a broad sense was reported for all the studied characters and medium heritability in narrow sense was obtained for days to $50 \%$ tasselling and to $50 \%$ silking, grain yield/plant, kernels/ row and 100- kernel weight, whereas low heritability in narrow sense was observed for plant height, flag leaf area, ear height, rows/ear, ear length and kernels/ear. The excepted genetic advance from selection was high for number of kernels/ear, grain yield/plant, flag leaf area and the value was $27.18,24.30$ and 18.55 , respectively. The excepted genetic advance was high as a percent for grain yield/plant and the value reached $20.50 \%$. These results are in agreement with studies of Deletic et al. (2005); Cook and Hallauer (2008) Hamed (2008); and Najeeb et al. (2009). Table (6) presents estimates of heterosis for all the studied characters. The hybrid $(2 \times 4)$ gave high value of heterosis for plant height, while the hybrid $(3 \times 4)$ and hybrid $(2 \times 4)$ gave negative heterosis for days to $50 \%$ tasselling and silking. But in flag leaf area the hybrids $(2 \times 5)$ and $(1 \times 5)$ gave high heterosis $(100 \%)$ for each of them. The hybrid $(2 \times 3)$ and hybrid $(4 \times 5)$ gave high value of heterosis for grain yield/plant (42.66 and $49.33 \%$, respectively). The other characters gave low value of heterosis in number of kernels/row, number of rows/ear, ear length and 100- kernels weight, while the number of kernels/ear gave high heterosis in hydrids $(4 \times 5),(3 \times 5),(2 \times 5),(2 \times 4),(2 \times 3)$ and $(1 \times 3)$, when compared with the heterosis that was calculated in relation to high parent. The same table showed that high heterosis was observed in hybrid $(4 \times 3)$ for traits plant height, flag leaf area, ear height, grain yield/plant and the number of kernels/row and also the value of heterosis was high for number of kernels/ear in hybrids $(2 \times 3),(2 \times 4),(3 \times 4)$ and hybrid $(3 \times 5)$. These results are in accordance with those of Chungji These results are in accordance with 
Table (3): Estimates of general and specific combining ability effects of parents and hybrids for studied characters.

\begin{tabular}{|c|c|c|c|c|c|c|c|c|c|c|c|}
\hline Characters & $\begin{array}{c}\text { Plant } \\
\text { height } \\
\text { (cm) }\end{array}$ & $\begin{array}{c}\text { Days to } 50 \\
\% \\
\text { tasselling }\end{array}$ & $\begin{array}{c}\text { Days to } \\
50 \% \\
\text { silking }\end{array}$ & $\begin{array}{c}\text { Flag leaf } \\
\text { area } \\
\left(\mathrm{cm}^{2)}\right.\end{array}$ & $\begin{array}{c}\text { Ear } \\
\text { height } \\
\text { (cm) }\end{array}$ & $\begin{array}{l}\text { Grain } \\
\text { yield } \\
\text { /plant } \\
\text { (g) }\end{array}$ & $\begin{array}{l}\text { Kernels / } \\
\text { row }\end{array}$ & $\begin{array}{c}\text { Rows/ } \\
\text { ear }\end{array}$ & $\begin{array}{c}\text { Ear } \\
\text { length } \\
(\mathrm{cm})\end{array}$ & $\begin{array}{c}100 \\
\text { Kernel } \\
\text { weight } \\
\text { (g) }\end{array}$ & $\begin{array}{c}\text { Kernels } \\
\text { / ear }\end{array}$ \\
\hline 1 & 4.33 & -0.21 & 0.33 & 19.23 & 0.46 & 7.14 & -1.09 & 0.62 & 0.38 & 1.13 & -2.11 \\
\hline 2 & -3.57 & -1.60 & -1.09 & -11.71 & -3.96 & 19.09 & 2.85 & -0.13 & 0.38 & 1.32 & 46.36 \\
\hline 3 & 5.76 & -0.45 & 0.09 & 19.23 & 2.89 & 4.33 & 0.04 & 0.43 & -0.04 & 0.08 & 11.88 \\
\hline 4 & -5.33 & 1.44 & 0.42 & -21.71 & 0.41 & -16.52 & -0.33 & -0.65 & -0.42 & -1.62 & -17.35 \\
\hline 5 & -1.19 & 0.82 & 0.23 & -5.04 & 0.18 & -14.04 & -1.43 & -0.27 & -0.28 & -0.91 & -38.78 \\
\hline $\operatorname{SE}\left(g_{i}-g_{k}\right)$ & 3.17 & 0.46 & 0.41 & 17.27 & 2.99 & 7.52 & 1.25 & 0.50 & 0.36 & 0.81 & 29.99 \\
\hline $1 \times 2$ & 20.31 & 10.79 & 10.26 & 59.68 & 6.88 & 35.06 & 4.85 & 1.82 & 3.15 & 6.41 & 80.85 \\
\hline $1 \times 3$ & -0.87 & 1.36 & 0.79 & 0.63 & 3.79 & 19.63 & 0.04 & 1.77 & 0.77 & 0.93 & 60.09 \\
\hline $1 \times 4$ & 4.22 & -1.87 & -1.20 & -31.74 & -3.73 & -8.50 & -1.57 & -2.46 & -0.17 & 1.65 & -124.00 \\
\hline $1 \times 5$ & 5.07 & -1.25 & -0.01 & 58.25 & 12.50 & 8.34 & 0.23 & 0.49 & -0.31 & 1.26 & 7.42 \\
\hline $2 \times 3$ & 1.03 & 2.07 & 1.88 & -25.07 & -1.77 & 21.34 & 2.42 & 0.53 & 0.11 & -0.25 & 53.61 \\
\hline $2 \times 4$ & 13.79 & -2.49 & -1.11 & 5.87 & 5.69 & -2.46 & 0.47 & 2.30 & -0.50 & -2.20 & 62.85 \\
\hline $2 \times 5$ & 1.98 & -1.53 & -0.92 & 55.87 & 0.93 & 5.73 & 4.28 & -0.07 & 1.34 & -0.92 & 36.95 \\
\hline $3 \times 4$ & -12.2 & -3.96 & -2.96 & -8.41 & -1.15 & 2.30 & -0.04 & 0.39 & -0.07 & 0.36 & 2.00 \\
\hline $3 \times 5$ & 15.31 & -0.01 & 0.88 & 11.58 & 6.41 & 1.82 & 1.09 & 0.01 & -0.55 & 0.31 & 25.42 \\
\hline $4 \times 5$ & 16.41 & -1.92 & 0.55 & 42.53 & 3.22 & 31.68 & 6.47 & 2.11 & 1.49 & -0.63 & 178.22 \\
\hline $\operatorname{SE}\left(s_{i j}-s_{i k}\right)$ & 7.77 & 1.14 & 1.01 & 42.31 & 7.34 & 18.44 & 3.06 & 1.23 & 0.90 & 1.99 & 73.46 \\
\hline
\end{tabular}


Table (4) : Estimates of additive $\left(\sigma^{2} A\right)$, dominance $\left(\sigma^{2} D\right)$, environmental $\left(\sigma^{2}\right.$ E) and phenotypic $\left(\sigma^{2} P\right)$ variances for studied characters.

\begin{tabular}{|c|c|c|c|c|c|c|c|c|c|c|c|}
\hline Variances & $\begin{array}{l}\text { Plant } \\
\text { height }\end{array}$ & $\begin{array}{c}\text { Days to } 50 \\
\% \\
\text { tasselling }\end{array}$ & $\begin{array}{c}\text { Days to } 50 \\
\% \\
\text { silking }\end{array}$ & $\begin{array}{c}\text { Flag leaf } \\
\text { area }\end{array}$ & $\begin{array}{c}\text { Ear } \\
\text { Height }\end{array}$ & $\begin{array}{l}\text { Grain } \\
\text { yield } \\
\text { /Plant }\end{array}$ & $\begin{array}{c}\text { Kernels / } \\
\text { row }\end{array}$ & $\begin{array}{l}\text { Rows/ } \\
\text { ear }\end{array}$ & $\begin{array}{c}\text { Ear } \\
\text { length }\end{array}$ & $\begin{array}{c}100 \\
\text { Kernel } \\
\text { weight }\end{array}$ & $\begin{array}{l}\text { Kernels } \\
\text { / ear }\end{array}$ \\
\hline$\sigma^{2} \mathrm{~A}$ & $\begin{array}{c}43.93 \\
27.32 \pm\end{array}$ & $\begin{array}{c}2.72 \\
1.61 \pm\end{array}$ & $\begin{array}{c}0.722 \\
0.45 \pm\end{array}$ & $\begin{array}{c}587.74 \\
397.58 \pm\end{array}$ & $\begin{array}{c}9.25 \\
7.11 \pm\end{array}$ & $\begin{array}{c}433.50 \\
261.23 \pm\end{array}$ & $\begin{array}{c}5.30 \\
3.36 \pm\end{array}$ & $\begin{array}{c}0.47 \\
0.32 \pm\end{array}$ & $\begin{array}{c}0.23 \\
0.16 \pm\end{array}$ & $\begin{array}{c}3.04 \\
1.88 \pm\end{array}$ & $\begin{array}{c}1750.26 \\
1186.14 \pm\end{array}$ \\
\hline$\sigma^{2} \mathrm{D}$ & $\begin{array}{l}140.59 \\
62.27 \pm\end{array}$ & $\begin{array}{c}7.10 \\
3.00 \pm\end{array}$ & $\begin{array}{c}2.18 \\
0.97 \pm\end{array}$ & $\begin{array}{c}1475.25 \\
749.80 \pm\end{array}$ & $\begin{array}{c}35.45 \\
18.95 \pm\end{array}$ & $\begin{array}{c}353.19 \\
172.03 \pm\end{array}$ & $\begin{array}{l}11.10 \\
5.29 \pm\end{array}$ & $\begin{array}{c}2.32 \\
1.07 \pm\end{array}$ & $\begin{array}{c}0.73 \\
0.36 \pm\end{array}$ & $\begin{array}{c}2.56 \\
1.37 \pm\end{array}$ & $\begin{array}{c}7946.14 \\
3682.43 \pm\end{array}$ \\
\hline$\sigma^{2} \mathrm{E}$ & $\begin{array}{l}35.28 \\
9.11 \pm\end{array}$ & $\begin{array}{c}0.76 \\
0.19 \pm \\
\end{array}$ & $\begin{array}{c}0.60 \\
0.15 \pm \\
\end{array}$ & $\begin{array}{r}1044.39 \\
269.66 \pm\end{array}$ & $\begin{array}{c}31.49 \\
8.13 \pm \\
\end{array}$ & $\begin{array}{c}198.38 \\
51.22 \pm\end{array}$ & $\begin{array}{c}5.47 \\
1.41 \pm \\
\end{array}$ & $\begin{array}{c}0.88 \\
0.22 \pm \\
\end{array}$ & $\begin{array}{c}0.47 \\
0.12 \pm \\
\end{array}$ & $\begin{array}{c}2.31 \\
0.59 \pm \\
\end{array}$ & $\begin{array}{l}3148.46 \\
812.9 \pm\end{array}$ \\
\hline$\sigma^{2} \mathrm{P}$ & 219.81 & 10.59 & 3.51 & 3107.39 & 76.20 & 985.08 & 21.88 & 3.68 & 7.92 & 7.92 & 12844.87 \\
\hline
\end{tabular}

Table (5): The average degree of dominance $(\overline{\mathbf{a}})$, heritability in broad sense $\left(\mathrm{h}_{\cdot b_{\mathrm{b} .} \cdot}\right)$, and narrow sense $\left(\mathrm{h}_{\bullet_{\mathrm{n} . \mathrm{s}}}\right)$ and expected genetic advance(GA) from selection for studied characters.

\begin{tabular}{|c|c|c|c|c|c|c|c|c|c|c|c|}
\hline $\begin{array}{l}\text { Characters } \\
\text { Genetic } \\
\text { parameters }\end{array}$ & $\begin{array}{l}\text { Plant } \\
\text { height }\end{array}$ & $\begin{array}{c}\text { Days to } 50 \\
\% \\
\text { tasselling }\end{array}$ & $\begin{array}{l}\text { Days to } \\
50 \% \\
\text { silking }\end{array}$ & $\begin{array}{c}\text { Flag leaf } \\
\text { area }\end{array}$ & $\begin{array}{c}\text { Ear } \\
\text { height }\end{array}$ & $\begin{array}{c}\text { Grain } \\
\text { yield } \\
\text { /Plant }\end{array}$ & $\begin{array}{c}\text { Kernels / } \\
\text { row }\end{array}$ & $\begin{array}{c}\text { Rows/ } \\
\text { ear }\end{array}$ & $\begin{array}{c}\text { Ear } \\
\text { length }\end{array}$ & $\begin{array}{c}100 \\
\text { Kernel } \\
\text { weight }\end{array}$ & $\begin{array}{c}\text { Kernels } \\
\text { / ear }\end{array}$ \\
\hline$\overline{\mathrm{a}}$ & 2.52 & 2.28 & 2.46 & 2.24 & 2.76 & 1.27 & 2.04 & 3.13 & 2.50 & 1.29 & 3.01 \\
\hline$h_{\text {.b.s. }}$ & 83.94 & 92.79 & 82.80 & 66.39 & 58.67 & 79.86 & 74.99 & 75.83 & 66.82 & 70.76 & 75.48 \\
\hline $\mathrm{h}_{\text {.n.s. }}$ & 19.98 & 25.74 & 20.55 & 18.91 & 12.14 & 44.00 & 24.24 & 12.80 & 16.16 & 38.42 & 13.61 \\
\hline GA & 5.21 & 1.47 & 0.67 & 18.55 & 1.86 & 24.30 & 1.99 & 0.43 & 0.34 & 1.90 & 27.18 \\
\hline GA \% & 3.67 & 2.16 & 0.97 & 2.88 & 2.68 & 20.50 & 5.75 & 2.62 & 2.24 & 9.13 & 4.73 \\
\hline
\end{tabular}


Table (6) : Heterosis (\%) relative to the mid - parent and high parent for studied characters.

\begin{tabular}{|c|c|c|c|c|c|c|c|c|c|c|c|}
\hline Characters & $\begin{array}{l}\text { Plant } \\
\text { height }\end{array}$ & $\begin{array}{c}\text { Days to } 50 \\
\% \\
\text { tasselling }\end{array}$ & $\begin{array}{l}\text { Days to } \\
50 \% \\
\text { silking }\end{array}$ & $\begin{array}{c}\text { Flag leaf } \\
\text { area }\end{array}$ & $\begin{array}{c}\text { Ear } \\
\text { height }\end{array}$ & $\begin{array}{c}\text { Grain } \\
\text { yield } \\
\text { /plant }\end{array}$ & $\begin{array}{c}\text { Kernels / } \\
\text { row }\end{array}$ & $\begin{array}{c}\text { Rows/ } \\
\text { ear }\end{array}$ & $\begin{array}{c}\text { Ear } \\
\text { length }\end{array}$ & $\begin{array}{c}100 \text { - } \\
\text { Kernel } \\
\text { weight }\end{array}$ & $\begin{array}{c}\text { Kernels } \\
\text { / ear }\end{array}$ \\
\hline & \multicolumn{11}{|c|}{ Heterosis relative to mid-parent } \\
\hline $1 \times 2$ & $10.00 *$ & $1.33^{*}$ & 0.83 & -26.66 & 1.83 & $34.33 * *$ & 0.83 & 0.16 & $1.83 * *$ & 4.66 & 18.66 \\
\hline $1 \times 3$ & 2.66 & 1.16 & 1.00 & -5.00 & $8.33^{*}$ & $39.66^{* *}$ & 0.50 & $2.33 * *$ & $1.16^{*}$ & 3.00 & $80.33^{*}$ \\
\hline $1 \times 4$ & $12.50 * *$ & $-4.5 * *$ & $-2.33 * *$ & -30.00 & 0.000 & 6.00 & -0.66 & $-2.00 * *$ & 0.33 & 3.16 & $-109.33 * *$ \\
\hline $1 \times 5$ & $17.50^{* * *}$ & $-2.5^{*}$ & 0.16 & $100.00 * *$ & $21.00 * *$ & $29.00^{* *}$ & 2.83 & 1.00 & 0.50 & 8.00 & 54.33 \\
\hline $2 \times 3$ & 6.66 & $1.83^{*}$ & $2.16^{* *}$ & -28.33 & 0.83 & $42.66^{* *}$ & $5.00 * *$ & $1.83^{*}$ & 0.66 & -0.60 & $126.33^{* *}$ \\
\hline $2 \times 4$ & $24.16^{* *}$ & $-5.16^{* *}$ & $-2.16^{* *}$ & 10.00 & 7.50 & 13.33 & 3.50 & $3.50 * *$ & 0.16 & $-2.50 *$ & $130.00^{* *}$ \\
\hline $2 \times 5$ & $16.5^{* *}$ & $-2.83^{*}$ & -0.66 & $100.00 * *$ & 7.50 & $27.66^{* *}$ & $9.00 * *$ & 1.16 & $2.33 * *$ & -1.00 & $136.00 * *$ \\
\hline $3 \times 4$ & -5.83 & $-6.66^{* *}$ & $-4.00 * *$ & -11.66 & 1.66 & $19.33^{*}$ & 2.16 & $1.66^{*}$ & 0.16 & 0.50 & 67.00 \\
\hline $3 \times 5$ & $25.83^{* *}$ & $-1.33^{*}$ & $1.16^{*}$ & $48.33^{*}$ & $14.00^{* * *}$ & $25.00^{*}$ & $5.00 * *$ & 1.33 & 0.00 & 0.66 & $122.66 * *$ \\
\hline \multirow[t]{2}{*}{$4 \times 5$} & $31.66^{* * *}$ & $-5.66^{* *}$ & -0.5 & $86.66^{* * *}$ & $10.00 *$ & $49.33 * *$ & $10.83 * *$ & $3.33 * *$ & 2.16 & -0.83 & $269.66 * *$ \\
\hline & \multicolumn{11}{|c|}{ Heterosis relative to high parent } \\
\hline $1 \times 2$ & 0.00 & $2.66 * *$ & $2.33 * *$ & $-60.00 *$ & -0.66 & $23.66^{*}$ & -1.00 & -1.33 & $1.66^{* *}$ & $2.66^{*}$ & 14.66 \\
\hline $1 \times 3$ & -0.66 & 1.33 & $1.33^{*}$ & -10.00 & 5.00 & $34.33 * *$ & 0.33 & 1.33 & 1.00 & $2.66 *$ & 44.00 \\
\hline $1 \times 4$ & 0.00 & $\begin{array}{l}-0.33 \\
\end{array}$ & $\begin{array}{l}-1.00 \\
\end{array}$ & -7.300 & -1.66 & -14.66 & -1.66 & $-4.00 * *$ & -0.33 & 2.33 & $-169.33^{* *}$ \\
\hline $1 \times 5$ & 5.00 & $\begin{array}{l}-0.33 \\
\end{array}$ & 0.33 & $93.33^{* * *}$ & $-59.33 * *$ & 4.66 & -1.00 & -0.66 & -0.33 & $2.66^{*}$ & -59.33 \\
\hline $2 \times 3$ & -6.66 & $3.00 * *$ & $3.33 * *$ & $-66.66^{*}$ & $94.00 * *$ & $26.66^{*}$ & 3.00 & 1.33 & 0.66 & -1.66 & $94.00 *$ \\
\hline $2 \times 4$ & $21.66^{* *}$ & 0.33 & 0.66 & 0.00 & $74.00 * *$ & $-18.00^{*}$ & 0.66 & $3.00 * *$ & -0.33 & -5.33 & 74.00 \\
\hline $2 \times 5$ & 14.00 ** & 0.66 & 0.66 & $66.66^{*}$ & $26.66^{* *}$ & -7.33 & 3.33 & 1.00 & $1.66 * *$ & -3.33 & 26.66 \\
\hline $3 \times 4$ & $-21.66 * *$ & $-2.33^{*}$ & $-2.33^{* *}$ & $-60.00 *$ & $43.33^{* *}$ & 4.00 & 1.33 & 0.66 & -0.33 & 1.66 & 43.33 \\
\hline $3 \times 5$ & $10.00 *$ & 1.00 & $1.33^{*}$ & -23.33 & $45.33^{* *}$ & 6.00 & 1.33 & 0.66 & -0.66 & 0.00 & 45.33 \\
\hline $4 \times 5$ & $31.66^{* * *}$ & $-3.66 * *$ & 1.00 & $63.33^{*}$ & 5.00 & $45.66^{* *}$ & $8.00 * *$ & $3.00 * *$ & $2.00 * *$ & -1.33 & $216.00^{* *}$ \\
\hline
\end{tabular}

$*$ and $* *$ indicate significant at 0.05 and 0.01 probability levels, respectively . 
those of Chungji et al. (2006) Lee et al. (2008), and Dawod et al. (2009).

From this study it is concluded that the parent (ZP-197) showed a high general combining ability in the number of traits and some hybrids showed high heterosis as well as high specific combining ability for many traits such as (DK $\times$ ZP-197) and (ZP-707× ZP-197) which can be of great benefit in the future breeding programs.

\section{REFERENCES}

Ahmed A. A. and Ali A. A. (2003). Estimation of several genetic parameters of maize (Zea mays L.). Iraqi J. Agric. Sci., 4 (3): $105-112$.

Al-Falahi M. A. H. (2000). Performance of some maize genotypes across location years and dates of planting. Iraqi J. Agric. Sci., 31 (2): 296 - 383.

Al-Jamili A. N. A. (2006). Heterosis, combining ability and some parameters in maize. Iraqi J. Agr. Sci., 37 (3) : 95106.

Al-Rawi K. M. and Khalaf - Allah A. M. (1980). Design and Analysis of Agricultural Experiments. Directorate of Book House of Publishing and Pressing, Mosul Univ., Iraq (In Arabic).

Al-Savie H. Sh. H. (2005). Gene action estimation of some parameters and heterosis in maize plant (Zea mays L.) by using factorial crosses. M. Sc. Thesis, Techniqual College - Almseape, Iraq.

Al-Sweediy M. H. Y. A. (2002). Full diallel estimation of genetic parameters, genetic and phenotypic correlation among characters for inbred lines of maize. Ph. D. Thesis, College of Agriculture, Baghdad University, Iraq (In Arabic).

Chungji H., Woongcho H. and Yamakawa T. (2006). Diallel analysis of plant and ear in tropical maize (Zea mays L.). J. Fac. Agr. Kyushu Univ., (51) (2) : $233-238$.

Cook K. A. and Hallauer A. R. (2008). Linkage disequilibrium in maize $F_{2}$ population of (B73× M17). Maize Genetic Newsletter, 82: 156-167.

Dawod K. M., Mohamad A.S. H and Kanosh Kh. H. (2009). Inheritance of grain yield in half diallel maize population. Iraqi J. Agric. Sci., in press.

Deletic N., Stokovic C., Duric V., Gudzic S. and Biberdzic M. (2005). The effect of high selection intensity on the change of maize yield components additive variance. Genetika, 37 (1): 71 - 76.

Derera J., Tongoona P., Vive B. S. and Laing M. D. (2007). Gene action controlling grain yield and secondary traits in southern African maize hybrids under drought and non- drought environments. Euphytica., $91: 89-97$.

Griffing B. (1956). Concept of general and specific combining ability in relation to diallel crossing systems. Aust. J. Biol. Sci., 9:463-493.

Hallauer A. R and Miranda J. B. (1988). Quantitative Genetics in Maize Breeding. $2^{\text {nd }}$ ed. Iowa State College Press, Ames. USA 468 pp.

Hamed M. H. Y. (2008). Estimation of some parameters for inbreds of maize (Zea mays L.) M. Sc. Thesis, Agric. College, Tikrite Univ., Iraq.

Lee E. A., Singh A., Ash M. J. and Good B. (2006). Use of sister lines and the performance of modified single - cross maize hybrids. Crop Sci., 46: $312-320$.

Mohammadi S. A., Prasanna B. M., Sudan C. and Singh N. N. (2008). SSR heterogenic patterns of maize parental lines and prediction of hybrid performance. Biotechnology and Biotechnological Equipment. 22 (1): $541-547$.

Najeeb S., Rather A. G., Parray G. A., Sheikh F. A. and Razvi S. M. (2009). Studies on genetic variability, genotypic correlation and path coefficient analysis in maize under high altitude temperate ecology of Kashmir. Maize Genetics Cooperation Newsletter, 83: $1-8$.

Ojo G. O. S., Adedzwa D. L. and Bello L. L. (2001). Combining ability estimates and heterosis for grain yield and yield components in maize (Zea mays L.). Makurdi, Nigeria

Paterniani M. E., Sawazaki A. G. Z, Gallo P. B., Luders R. R. and Silva R. M. (2004). Estimates of genetic parameters in maize composite and potential for recurrent selection. Crop Breeding and Applied Biotechnology, Brazilian Society of Plant Breeding. 4: $81-85$.

Rather A. G., Najeeb S., Sheikh F. A., Shikhari A. B. and Dar Z. A. (2007). Combining ability analysis in maize (Zea mays L.) under high altitude temperate 
conditions of Kashmir SK University of Agriculture Science and Technology of Kashmir Maize Genetic Cooperation Newsletter.81:http://www.agron.missour i.edu/mn1/81/42rather.htm

Rather A. G., Najeeb S., Wani A. A., Bhat M. A. and Parray G. A. (2009). Combining ability analysis for turcicum leaf blight (TIB) and other agronomic traits in maize (Zea mays $\mathrm{L}$ ) under high altitude temperate conditions of Kashmir Maize Genetic Cooperation Newsletter, 83: 1-5. Rezaéi A., Yazdisamadi B. and Zali A. (2004).
Estimate of heterosis and combining ability in maize (Zea mays L.) using diallel crossing method. Genetic Variation for Plant Breeding, p.395397. http://www.ctahr.hawaii

Singh R. K. and Chaudhary B. D. (2007). Biometrical Methods in Quantitative Genetic Analysis. Rew. Ed., Kalyani Publishers Ludhiana, India.

Sprague G. F. and Tatum L.A. (1942). General versus specific combining ability in single crosses of corn. J. Amer. Soc. Agron., 34: 923-93.

\section{التحليل الوراثي للتهجين التبادلي النصفي بين خمس سلالات تربية داخلية من الذرة الثامية متوسطة التبكير بالنضج خمالف

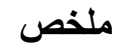

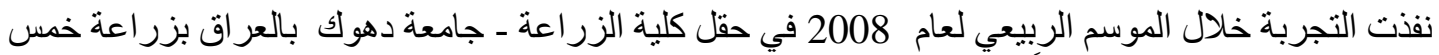

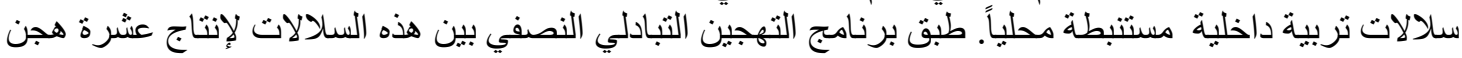

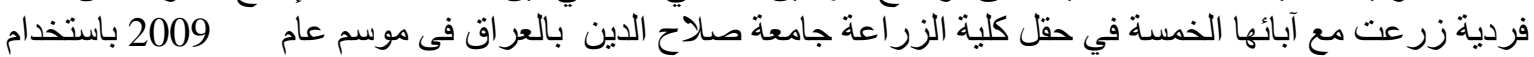

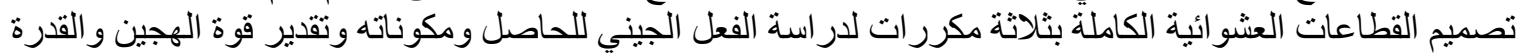

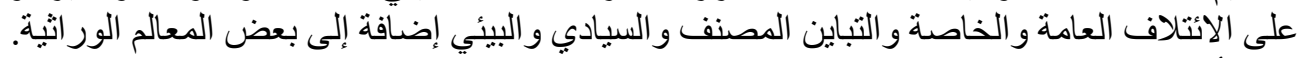

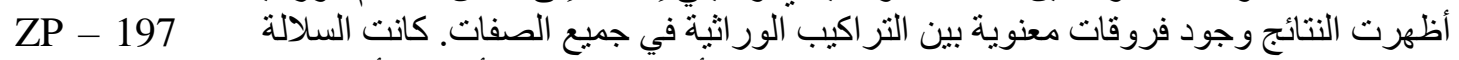

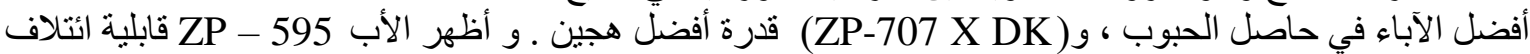

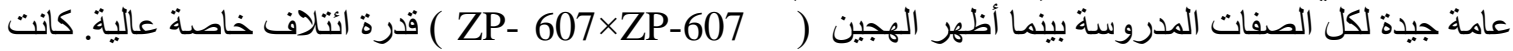

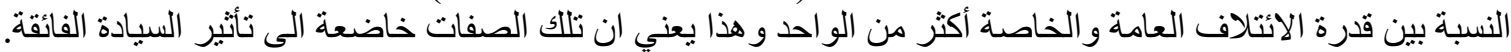

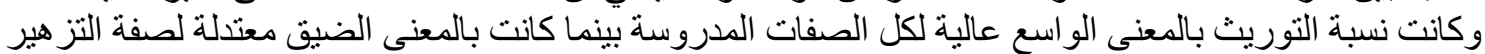

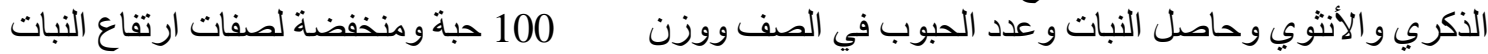

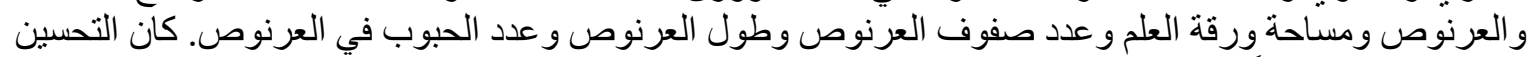

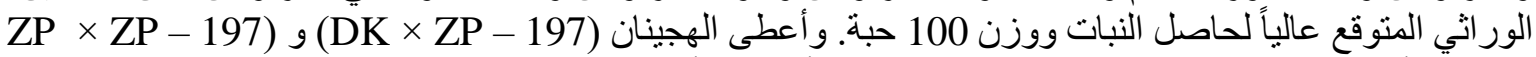

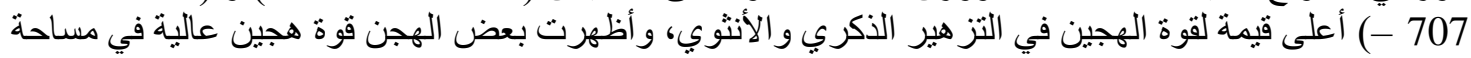

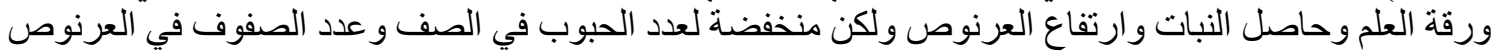
وطول العرنوص ووزن 100 حبة ولمختلف الهجن.

المجلة العلمية لكلية الزراعة - جامعة القاهرة ـ المجلا (61) العدد الرابع (اكتوبر 2010):354-346. 